\title{
Height and body fatness and colorectal cancer risk: an update of the WCRF-AICR systematic review of published prospective studies
}

\author{
Leila Abar $^{1}$ - Ana Rita Vieira ${ }^{1}$. Dagfinn Aune ${ }^{1} \cdot$ Jakub G. Sobiecki $^{1}$. \\ Snieguole Vingeliene ${ }^{1} \cdot$ Elli Polemiti $^{1} \cdot$ Christophe Stevens $^{1} \cdot$ Darren C. Greenwood $^{2}$ • \\ Doris S. M. Chan ${ }^{1} \cdot$ Sabrina Schlesinger $^{1} \cdot$ Teresa Norat $^{1}$
}

Received: 29 November 2016 / Accepted: 25 June 2017 / Published online: 28 October 2017

(C) The Author(s) 2017. This article is an open access publication

\begin{abstract}
Purpose There is no published dose-response meta-analysis on the association between height and colorectal cancer risk (CRC) by sex and anatomical sub-site. We conducted a meta-analysis of prospective studies on the association between height and CRC risk with subgroup analysis and updated evidence on the association between body fatness and CRC risk.

Methods PubMed and several other databases were searched up to November 2016. A random effects model was used to calculate dose-response summary relative risks (RR's).

Results 47 studies were included in the meta-analyses including 50,936 cases among 7,393,510 participants. The findings support the existing evidence regarding a positive association of height, general and abdominal body fatness and CRC risk. The summary RR were 1.04 [95\% (CI)1.02$1.05, I^{2}=91 \%$ ] per $5 \mathrm{~cm}$ increase in height, 1.02 [95\% (CI) $1.01-1.02, I^{2}=0 \%$ ] per $5 \mathrm{~kg}$ increase in weight, 1.06 [95\% (CI) $\left.1.04-1.07, R^{2}=83 \%\right]$ per $5 \mathrm{~kg} / \mathrm{m}^{2}$ increase in BMI, 1.02 [95\% (CI) $\left.1.02-1.03, I^{2}=4 \%\right]$ per $10 \mathrm{~cm}$ increase in waist circumference, 1.03 [95\% (CI) $1.01-1.05, I^{2}=16 \%$ ] per 0.1 unit increase in waist to hip ratio. The significant
\end{abstract}

Electronic supplementary material The online version of this article (doi:10.1007/s00394-017-1557-1) contains supplementary material, which is available to authorized users.

Leila Abar

1.abar@imperial.ac.uk

1 Department of Epidemiology and Biostatistics, School of Public Health, Imperial College London, St. Mary's Campus, Norfolk Place, Paddington, London W2 1PG, UK

2 Biostatistics Unit, Centre for Epidemiology and Biostatistics, University of Leeds, Leeds, UK association for height and CRC risk was similar in men and women. The significant association for BMI and CRC risk was stronger in men than in women.

Conclusion The positive association between height and risk of CRC suggests that life factors during childhood and early adulthood might play a role in CRC aetiology. Higher general and abdominal body fatness during adulthood are risk factors of CRC and these associations are stronger in men than in women.

Keywords Height $\cdot \mathrm{BMI} \cdot$ Colorectal cancer $\cdot$ Metaanalysis $\cdot$ Continuous update project

\section{Introduction}

Colorectal cancer (CRC) is the second most common cancer in women and the third most common cancer in men with 614,000 new cases diagnosed among women and 746,000 cases in men worldwide in 2012. It is a leading cause of cancer-related death, resulting in around 700,000 deaths worldwide [1].

In the World Cancer Research Fund (WCRF)/American Institute for Cancer Research report from 2011, it was stated that the evidence that greater adult attained height increases colorectal cancer risk was convincing, based on the results from eight studies [2]. Since then, ten additional large cohort studies have been published on height and colorectal cancer [3-11]. In addition, Mendelian Randomization studies have suggested a causal association between height and colorectal cancer $[12,13]$, however, one of these found an association only among women [12]. No previous metaanalyses have examined the shape of the dose-response relationship between height and colorectal cancer, and the most recent meta-analysis did not investigate whether the 
association persisted in subgroup analyses stratified by study characteristics.

Adiposity is also an established risk factor for colorectal cancer, both as measured by body mass index (BMI) and waist circumference and waist-to-hip ratio. Although many studies have investigated the association between BMI and colorectal cancer [8, 9, 14-40], fewer studies have been published on abdominal fat measures and colorectal cancer risk. Although the World Cancer Research Fund (WCRF)/Continuous Update Project (CUP) 2011 report [2] concluded that there was convincing evidence for an association between both general and abdominal fatness and colorectal cancer, the analyses for waist circumference and colorectal, colon and rectal cancer were based on only 3, 6 and 3 studies, respectively [24, 27, 41-46]. Previous meta-analyses [47, 48] have not investigated the shape of the dose-response relationship between BMI, waist circumference or waist-tohip ratio and colorectal cancer $[47,48]$. New studies, that could have been included in our meta-analysis, have been published on BMI $(n=24)$ [8, 9, 11, 14, 15, 17-24, 49-51], waist circumference $(n=13)$ [9, 16, 18, 22-24, 52, 53] and waist-to-hip ratio $(n=6)[9,16,18,49,53]$ and colorectal cancer since the WCRF/CUP 2011 report and for this reason we conducted an updated meta-analysis of the available evidence from prospective studies. We aimed to clarify the strength and shape of the dose-response relationship between height, weight, BMI, waist circumference, and waist-to-hip ratio and colorectal cancer risk and to clarify any potential differences by sex, and geographical location.

\section{Materials and methods}

\section{Search strategy}

PubMed, Embase, CABAbstracts, ISI Web of Science, BIOSIS, LILACS, Cochrane library, CINAHL, AMED, National Research Register and In Process Medline were searched for studies on anthropometric measures including BMI, height, weight, waist circumference and waist to hip ratio, and colorectal cancer risk up to December 2015. The specific search criteria and the review protocol can be found at: http://www.wcrf.org/int/researchwe-fund/continuous-update-project-findings-reports/ colorectal-bowel-cancer.

\section{Study selection}

The search was restricted to cohort (prospective, retrospective, case-cohort or nested case-control studies) studies which investigated the link between anthropometric measures and colorectal cancer risk and reported estimates of the relative risk (RR) (e.g., hazard ratio, risk ratio or odds ratio) and 95\% confidence intervals (CIs) for the exposures of interest (BMI, height, weight, waist circumference, and waist-to-hip ratio), total number of cases and person years of follow-up. If there were multiple publications from the same study, the newest publication which included the largest number of cases was selected.

\section{Data extraction}

From each publication, the following data were extracted: first author's last name, year of publication, the study name, period of follow-up, sample size, age, sex, number of cases, country where the study was conducted, assessment method of exposure (self-reported vs measured), anthropometric measures, their quantities and their associated RRs and 95\% CIs, and variables used in adjustment in the analysis. The update search and data extraction from January 2010 up to November 2016 was conducted by three authors (LA, AR, JGS) and was checked for accuracy by two authors (TN, LA).

\section{Statistical methods}

The associations between height, weight, BMI, waist circumference and waist-to-hip ratio and colorectal, colon and rectal cancer were investigated using random effect models to calculate the summary RRs and 95\% CIs to take into account heterogeneity across studies [54]. Q and $I^{2}$ statistics were used to determine heterogeneity [55] and were explored in stratified analyses. Low proportion of heterogeneity across studies was defined by an $I^{2}<30 \%$, moderate proportion by an $I^{2}=30-50 \%$, and high proportion by an $I^{2} \geq 50 \%$.

Continuous risk estimates were used directly when available in the articles, and for studies that only reported categorical data dose-response associations and 95\% CIs were derived using generalized least-squares for trend estimation [56], which required the RRs and CIs associated to at least three categories of anthropometric measures, and number of cases and non-cases or person years of follow-up per category to be available. If only the total number of cases or person years was reported in the articles, and the exposure was categorised in quantiles, the distribution of cases or person years was calculated by dividing the total number of cases or person years by the number of quantiles.

The mean or median values per category were used if provided in the articles, or the midpoint was calculated for studies that only reported a range by category. If the range of the highest or lowest category was open-ended, its width was assumed to be the same as the adjacent category. If the results were reported for men and women separately, they were combined using a fixed effects meta-analysis before being pooled with other studies. 
Small-study effects, such as publication bias, were assessed using funnel plots and Egger's test [57].

A potential nonlinear dose-response association between anthropometric measures and colorectal cancer risk was assessed by calculating restricted cubic splines for each study with more than three categories of exposure, using three fixed knots at 10,50, and 90\% through the total distribution of the reported measurements, and combined them using multivariate meta-analysis. Indication of non-linearity was tested using likelihood ratio test.

For all analyses, the results of each paper with the most comprehensive adjustment for confounders were included. A two-tailed $p<0.05$ was considered statistically significant.

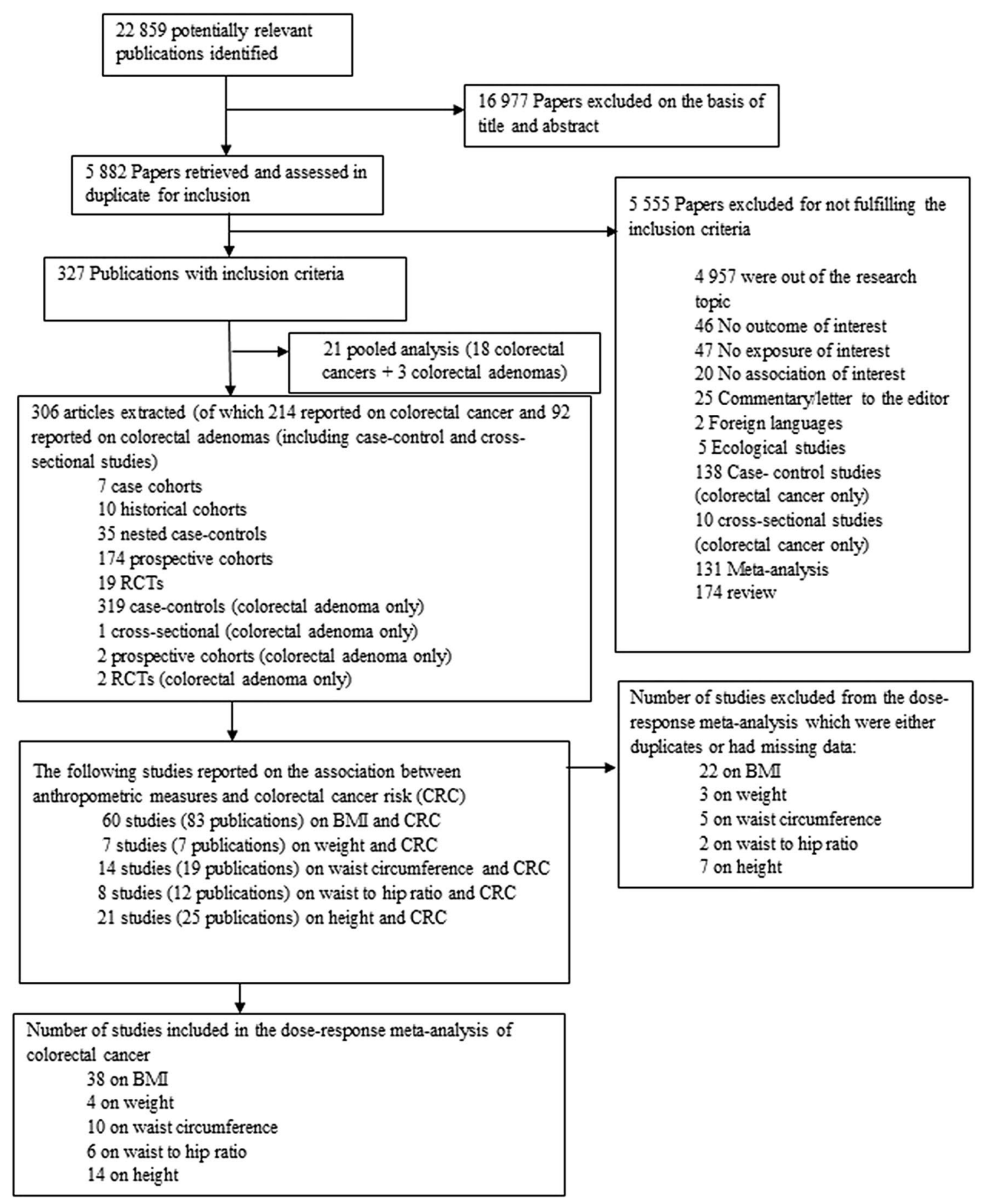

Fig. 1 Flowchart of study selection 
A Height and colorectal cancer, per $5 \mathrm{~cm}$

\begin{tabular}{|c|c|c|c|}
\hline Author & Year & $\begin{array}{l}\text { per } 5 \text { cm } \\
\text { RR }(95 \% \mathrm{Cl})\end{array}$ & $\begin{array}{l}\% \\
\text { Weigl }\end{array}$ \\
\hline Burón Pust & 2017 & $1.01(1.00,1.01)$ & 20.43 \\
\hline Boursi & 2014 & $1.06(1.04,1.08)$ & 14.98 \\
\hline Kabat* & 2013 & $1.06(1.00,1.11)$ & 4.80 \\
\hline Kabat*z & 2013 & $1.09(1.05,1.13)$ & 7.83 \\
\hline Walter & 2013 & H $1.12(0.94,1.32)$ & 0.58 \\
\hline Hughes & 2011 & $1.03(0.97,1.08)$ & 4.61 \\
\hline Oxentenko & 2010 & $1.01(1.00,1.01)$ & 20.09 \\
\hline Bowers & 2006 & $1.01(0.92,1.11)$ & 1.77 \\
\hline Engeland & 2005 & $1.05(1.04,1.06)$ & 18.45 \\
\hline Otani & 2005 & $1.03(0.95,1.10)$ & 275 \\
\hline Gunnell & 2003 & $0.97(0.73,1.28)$ & 0.22 \\
\hline Hebert & 1997 & $1.05(0.97,1.15)$ & 211 \\
\hline Kato & 1997 & $1.01(0.84,1.20)$ & 0.52 \\
\hline Albanes & 1988 & $+1.18(1.03,1.36)$ & 0.85 \\
\hline \multicolumn{2}{|c|}{ Overall (1-squared $=91.0 \%, p=0.000)$} & $1.04(1.02,1.05)$ & 100.0 \\
\hline \multicolumn{4}{|c|}{ NOTE: Weights are from random effects analy gis } \\
\hline & .75 & 1.3 & \\
\hline
\end{tabular}

B Height and colorectal cancer, nonlinear dose-response

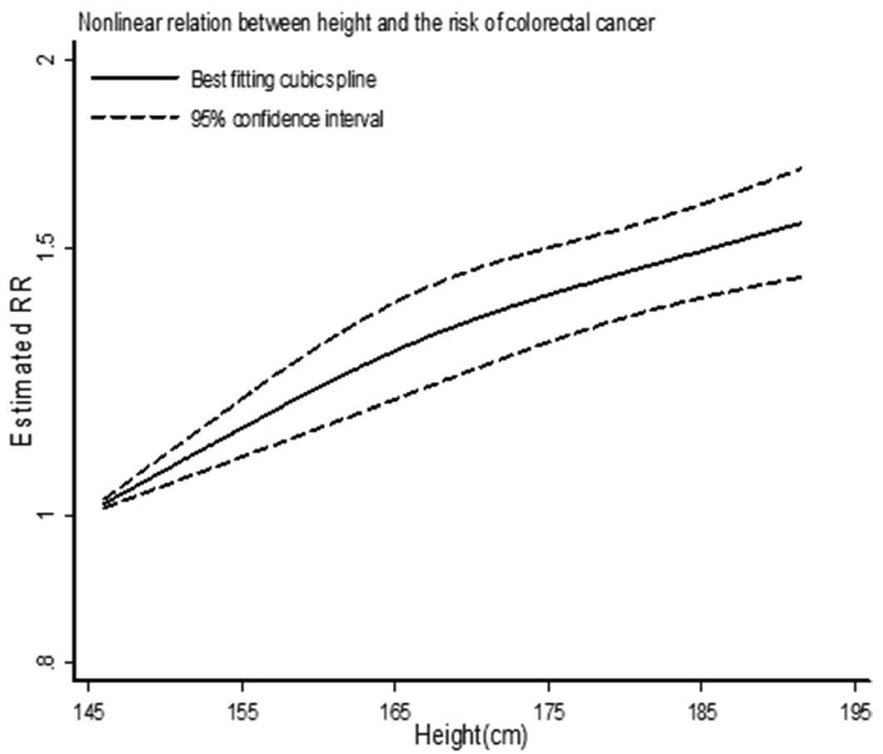

"Kabat, 2013 is Canadian National Breast Screening Study. ${ }^{*}$ Kabat. 2013 is Women Health Initiative Study.

Fig. 2 Height and colorectal cancer risk (dose-response and nonlinear analysis). $R R$ relative risk, 95\% CI 95\% confidence interval. Summary RR calculated using a random-effects model

Stata version 12 software (StataCorp, College Station, TX, USA) was used.

\section{Results}

In total, 47 studies including 50,960 cases among 7,393,510 participants were included in the meta-analysis of anthropometric measures and colorectal, colon (proximal and distal), and rectal cancer risk (flowchart of study selection-Fig. 1). Characteristics of the included studies are provided in Supplemental Table 1.

\section{Height}

\section{Height and colorectal cancer}

Fourteen studies $(84,095$ cases $)$ were included in the dose-response meta-analysis $[3,5,6,8,9,11,26,30$, 58-63]. The summary RR for an increase of $5 \mathrm{~cm}$ was 1.04 (95\% CI 1.02-1.05) (Fig. 2a). There was high heterogeneity $\left(I^{2}=91 \%, P_{\text {heterogeneity }}<0.001\right)$ and evidence of publication or small-study bias $(P$ value Egger's test $<0.05)$.

In stratified analysis the associations were similar in men and women and were stronger in studies in North
America compared to studies in Europe. In addition, the associations tended to be stronger in studies in which height was measured than self-reported and in studies with less than 10 years of follow-up (Table 1).

No evidence of nonlinear association was observed $\left(P_{\text {nonlinearity }}=0.33, n=10\right)($ Fig. $2 \mathrm{~b})$.

\section{Height and colon cancer}

Fourteen studies $(92,069$ cases) were included in the dose-response meta-analysis $[4,5,7,26,30,33,41,45$, $58,64-67]$. The summary RR for an increase of $5 \mathrm{~cm}$ was 1.02 (95\% CI 1.02-1.03). There was high heterogeneity $\left(I^{2}=87 \%, P_{\text {heterogeneity }}<0.001\right)$ (Table 1 , Supplemental Fig. 1A). There was evidence of publication or small study bias ( $P$ value Egger's test $<0.001$ ).

In stratified analysis by sex and geographical location, the summary RR showed similar association in studies in men and women and stronger association in studies in North America than studies in Europe. No significant association was observed in studies in Asia including two studies (Table 1).

There was evidence of a significant non-linear association $\left(P_{\text {nonlinearity }}=0.03, n=9\right)$, showing a significant 
Table 1 Summary of results

Height, per $5 \mathrm{~cm}$

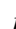

Colorectal cancer

All studies

14

Stratified by sex

\section{Men}

Women

Stratified by geographic location

Europe

North America

Asia

Australia

Duration of follow-up

$<10$ years follow-up

$10-<15$ years follow-up

$\geq 15$ years follow-up

Assessment of height

Measured

Self-reported

Number of cases

Cases $<1000$

Cases 1000-3000

Cases $\geq 3000$

Colon cancer

All studies

Stratified by sex

Men

Women

Stratified by geographic location

Europe

North America

Asia

Australia

Proximal colon cancer

All studies

Stratified by sex

Men

Women

Stratified by geographic location

Europe

North America

Asia

Australia

Distal colon cancer

All studies

Stratified by sex

Men

Women

Stratified by geographic location

Europe

10

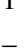

RR (95\% CI)

$I^{2}(\%)$

$P_{\mathrm{h}}$

1.04 (1.02-1.05)

91

$<0.001$

$1.04(1.03-1.05)$

$1.04(1.02-1.05)$

0

0.46

$1.03(1.00-1.07)$

91.5

$<0.001$

95.6

$<0.001$

$1.06(1.01-1.11)$

79.7

$<0.001$

$1.03(0.95-1.10)$

$-$

$-$

$-$

$1.06(1.04-1.08)$

$1.05(1.00-1.11)$

$1.03(1.00-1.07)$

$1.05(1.04-1.06)$

$1.01(1.00-1.02)$

$1.05(1.00-1.09)$

$1.04(1.00-1.09)$

$1.04(1.00-1.08)$

$1.02(1.02-1.03)$

$1.02(1.01-1.04)$

$1.02(1.01-1.03)$

$1.01(1.00-1.02)$

1.05 (1.01-1.10)

$1.12(0.96-1.32)$

$1.13(1.04-1.23)$

$1.02(0.99-1.05)$

$1.12(0.90-1.40)$

$1.01(0.99-1.03)$

$1.03(0.96-1.11)$

$1.01(1.00-1.01)$

$-$

$1.24(1.11-1.39)$

$1.01(1.00-1.01)$

$1.05(0.92-1.19)$

$1.01(1.00-1.02)$

$1.05(0.94-1.17)$ 
Table 1 (continued)

\begin{tabular}{|c|c|c|c|c|}
\hline & \multicolumn{4}{|c|}{ Height, per $5 \mathrm{~cm}$} \\
\hline & $n$ & $\mathrm{RR}(95 \% \mathrm{CI})$ & $I^{2}(\%)$ & $P_{\mathrm{h}}$ \\
\hline North America & 2 & $1.01(1.00-1.01)$ & 0 & 0.58 \\
\hline Asia & - & - & - & - \\
\hline Australia & 1 & $1.04(0.87-1.24)$ & & \\
\hline \multicolumn{5}{|l|}{ Rectal cancer } \\
\hline All studies & 14 & $1.01(1.00-1.02)$ & 61.7 & 0.002 \\
\hline \multicolumn{5}{|l|}{ Stratified by sex } \\
\hline Men & 10 & $1.02(1.00-1.05)$ & 39.7 & 0.09 \\
\hline Women & 12 & $1.01(1.00-1.01)$ & 57.8 & 0.006 \\
\hline \multicolumn{5}{|c|}{ Stratified by geographic location } \\
\hline Europe & 6 & $1.00(1.00-1.01)$ & 37.0 & 0.16 \\
\hline North America & 5 & $1.06(1.02-1.10)$ & 28.5 & 0.24 \\
\hline Asia & 2 & $1.04(1.00-1.09)$ & 0 & 0.61 \\
\hline \multirow[t]{3}{*}{ Australia } & - & - & - & - \\
\hline & \multicolumn{4}{|c|}{ Weight, per $5 \mathrm{~kg}$} \\
\hline & $n$ & $\mathrm{RR}(95 \% \mathrm{CI})$ & $I^{2}(\%)$ & $P_{\mathrm{h}}$ \\
\hline \multicolumn{5}{|l|}{ Colorectal cancer } \\
\hline All studies & 4 & $1.02(1.01-1.02)$ & 0 & 0.58 \\
\hline \multicolumn{5}{|l|}{ Stratified by sex } \\
\hline Men & 2 & $1.01(1.00-1.02)$ & 0 & 0.59 \\
\hline Women & 1 & $1.02(1.01-1.03)$ & - & - \\
\hline \multicolumn{5}{|c|}{ Stratified by geographic location } \\
\hline Europe & 3 & $1.02(1.01-1.03)$ & - & - \\
\hline North America & 1 & $1.02(1.01-1.03)$ & - & - \\
\hline Asia & - & - & - & - \\
\hline Australia & - & - & - & - \\
\hline \multicolumn{5}{|l|}{ Duration of follow-up } \\
\hline$<10$ years follow-up & 1 & $1.02(1.00-1.03)$ & - & - \\
\hline $10-<15$ years follow-up & 1 & $1.01(1.00-1.03)$ & - & - \\
\hline$\geq 15$ years follow-up & 2 & $1.02(1.01-1.03)$ & 5.7 & 0.30 \\
\hline \multicolumn{5}{|l|}{ Assessment of weight } \\
\hline Measured & 2 & $1.02(0.99-1.05)$ & 35.5 & 0.21 \\
\hline Self-reported & 2 & $1.02(1.01-1.03)$ & 0 & 0.88 \\
\hline \multicolumn{5}{|l|}{ Number of cases } \\
\hline Cases $<1000$ & 3 & $1.02(1.01-1.03)$ & 0 & 0.43 \\
\hline Cases 1000-3000 & - & - & - & - \\
\hline Cases $\geq 3000$ & - & - & - & - \\
\hline \multicolumn{5}{|l|}{ Colon cancer } \\
\hline All studies & 6 & $1.02(1.01-1.03)$ & 18 & 0.29 \\
\hline \multicolumn{5}{|l|}{ Stratified by sex } \\
\hline Men & 4 & $1.02(1.01-1.02)$ & 0 & 0.68 \\
\hline Women & 2 & $1.05(1.01-1.09)$ & 6 & 0.30 \\
\hline \multicolumn{5}{|c|}{ Stratified by geographic location } \\
\hline Europe & 3 & $1.01(1.00-1.02)$ & 0 & 0.92 \\
\hline North America & 1 & $1.03(1.01-1.05)$ & - & - \\
\hline Asia & - & - & - & - \\
\hline Australia & 2 & $1.05(1.01-1.09)$ & 6 & 0.30 \\
\hline
\end{tabular}


Table 1 (continued)

$$
\text { Weight, per } 5 \mathrm{~kg}
$$

\begin{tabular}{llll}
\hline$n$ & RR $(95 \% \mathrm{CI})$ & $I^{2}(\%)$ & $P_{\mathrm{h}}$ \\
\hline
\end{tabular}

Proximal colon cancer

All studies

\section{3}

Stratified by sex

Men

Women

1

2

Stratified by geographic location

Europe

North America

Asia

Australia

Distal colon cancer

All studies

Stratified by sex

Men

Women

Stratified by geographic location

Europe

North America

Asia

Australia

Rectal cancer

All studies

Stratified by sex

Men

Women

Stratified by geographic location

Europe

North America

Asia

Australia

Australia

4

1
$1.02(1.00-1.04)$

34

0.22

$1.01(0.98-1.04)$

$1.03(0.98-1.09)$

66.3

0.08

$1.01(0.98-1.04)$

$1.01(1.00-1.03)$

$-$

1.07 (1.01-1.14)

$1.03(1.01-1.05)$

23

0.27

$1.02(0.99-1.05)$

$1.05(0.99-1.11)$

$1.02(0.99-1.05)$

$1.02(1.01-1.04)$

$-$

1.09 (1.01-1.18)

$1.01(1.00-1.02)$

0

0.65

$1.01(1.00-1.02)$

0

0.78

$1.06(0.71-1.57)$

$1.01(1.00-1.02)$

$1.04(0.99-1.10)$

BMI, per $5 \mathrm{~kg} / \mathrm{m}^{2}$

n

Colorectal cancer

All studies

38

Stratified by sex

Men

Women

Stratified by geographic location

Europe

North America

Asia

Australia

Duration of follow-up

$<10$ years follow-up

$10-<15$ years follow-up

$\geq 15$ years follow-up
20

24

10

13

15

13

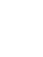

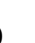

4

.05 (1.03-1.07)

$1.04(1.02-1.06)$

$1.05(1.03-1.07)$

1.09 (1.01-1.18)

$1.05(1.03-1.07)$
$1.06(1.03-1.09)$
$1.05(1.02-1.07)$

6

$\begin{array}{ll}83 & <0.001 \\ 83 & <0.001 \\ 83.7 & <0.001 \\ & \\ 80.8 & <0.001 \\ 61.2 & 0.003 \\ 86.2 & <0.001 \\ - & - \\ 33.7 & \\ 91.7 & 0.11 \\ 23.7 & <0.001 \\ \end{array}$


Table 1 (continued)

BMI, per $5 \mathrm{~kg} / \mathrm{m}^{2}$

$n \quad$ RR $(95 \% \mathrm{CI})$

\section{Assessment of weight/height}

Measured

25

Self-reported

Number of cases

Cases $<1000$

Cases 1000-3000

Cases $\geq 3000$

Colon cancer

All studies

Stratified by sex

Men

Women

Stratified by geographic location

Europe

North America

Asia

Australia

Proximal colon cancer

All studies

Stratified by sex

Men

Women

Stratified by geographic location

Europe

North America

Asia

Australia

Distal colon cancer

All studies

Stratified by sex

Men

Women

Stratified by geographic location

Europe

North America

Asia

Australia

Rectal Cancer

All studies

Stratified by sex

Men

Women

Stratified by geographic location

Europe

North America

Asia

Australia
13

22

4

11

42

26

30

13

14

1
1.05 (1.03-1.07)

1.06 (1.04-1.08)

$1.03(1.02-1.05)$

$1.06(1.03-1.09)$

$1.09(1.04-1.15)$

$1.07(1.05-1.08)$

$1.10(1.07-1.13)$

$1.04(1.02-1.05)$

$1.05(1.03-1.07)$

$1.08(1.05-1.11)$

$1.09(1.03-1.16)$

$1.13(1.00-1.28)$

$1.05(1.03-1.08)$

$1.13(1.05-1.21)$

1.04 (1.01-1.07)

$1.12(0.99-1.27)$

1.04 (1.02-1.07)

1.16(1.06-1.27)

$-$

$1.08(1.04-1.11)$

$1.23(1.08-1.38)$

1.05 (1.03-1.08)

$1.34(1.16-1.53)$

$1.05(1.03-1.08)$

$1.18(1.17-1.29)$

$-$

$1.02(1.01-1.03)$

$1.02(1.01-1.04)$

$1.01(0.99-1.03)$

$1.01(1.00-1.02)$

$1.02(0.99-1.05)$

$1.04(1.02-1.06)$

$1.02(0.97-1.08)$
$I^{2}(\%)$

28.1

0.17

85.9

$<0.001$

15.7

0.25

59.2

0.06

97.6

$<0.001$

78.8

$<0.001$

74.2

$<0.001$

57

$<0.01$

80.6

$<0.001$

75.8

$<0.001$

78.6

$<0.001$

$-$

44.0

0.04

33.2

0.20

30.4

0.17

33.1

51.6

0.01

77

$<0.01$

0

0.60

0

0.53

-

29.3

0.07

21.8

0.20

43.6

0.02

32.1

0.13

23.9

0.24

0

0.71

$-$ 
Table 1 (continued)

Waist circumference, per $10 \mathrm{~cm}$

Colorectal cancer

All studies

Stratified by sex

Men

Women

Stratified by geographic location

Europe

North America

Asia

Australia

Duration of follow-up

$<10$ years follow-up

$10-<15$ years follow-up

$\geq 15$ years follow-up

Assessment of waist circumference

Measured

Self-reported

Number of cases

Cases $<1000$

Cases 1000-3000

Cases $\geq 3000$

Colon cancer

All studies

Stratified by sex

Men

Women

Stratified by geographic location

Europe

North America

Asia

Australia

Proximal colon cancer

All studies

Stratified by sex

Men

Women

Stratified by geographic location

Europe

North America

Asia

Australia

Distal colon cancer

All studies

Stratified by sex

Men

Women

Stratified by geographic location

Europe $n$

10

RR (95\% CI)

$1.02(1.02-1.03)$

$1.03(0.99-1.06)$

$1.03(1.02-1.04)$

1.01 (0.99-1.04)

1.03 (1.01-1.04)

1.03 (1.01-1.05)

$-$

$1.02(1.01-1.04)$

$1.00(0.98-1.03)$

$1.06(0.98-1.14)$

1.02 (1.00-1.05)

$1.02(1.01-1.04)$

1.02 (1.01-1.03)

1.03 (1.01-1.04)

1

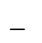

11

$1.05(1.02-1.07)$

$1.09(1.03-1.15)$

1.03 (1.01-1.04)

$1.02(1.01-1.04)$

1.04 (1.01-1.08)

1.05 (1.02-1.08)

2

$-$

5

2

2

$1.12(0.95-1.31)$

1.03 (1.01-1.05)

$1.04(0.98-1.10)$

$1.06(1.01-1.11)$

$-$

$-$

5

2

2

1

$1.06(1.02-1.11)$

$1.14(0.94-1.38)$

1.14 (1.01-1.06)

$1.05(0.98-1.11)$

$I^{2}(\%)$

$P_{\mathrm{h}}{ }^{1}$

4.2

0.40

77.9

0.001

0

0.90

34.4

0.22

30.7

0.23

0

0.79

0

0.97

0

69.5

0.65

0.07

0

0.76

40.7

0.15

12.9

0.33

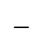

72

$<0.001$

95

$<0.001$

0.7

0.42

0

0.88

69.9

0.01

$-$

$-$

49.9

0.09

79

0.03

0

0.50

62.1

0.05

$-$

$-$

50.5

0.09

83.5

0.01

0

0.65 
Table 1 (continued)

\begin{tabular}{|c|c|c|c|c|}
\hline & & $\mathrm{r} 10 \mathrm{~cm}$ & & \\
\hline & $n$ & RR $(95 \% \mathrm{CI})$ & $I^{2}(\%)$ & $P_{\mathrm{h}}{ }^{1}$ \\
\hline North America & 4 & $1.07(1.01-1.14)$ & 62.8 & 0.04 \\
\hline Asia & - & - & - & - \\
\hline Australia & - & - & - & - \\
\hline Rectal cancer & & & & \\
\hline All studies & 8 & $1.03(1.00-1.05)$ & 45.7 & 0.10 \\
\hline Stratified by sex & & & & \\
\hline Men & 6 & $1.02(0.98-1.06)$ & 59.2 & 0.03 \\
\hline Women & 5 & $1.04(1.01-1.06)$ & 0 & 0.48 \\
\hline Stratified by geographic lo & & & & \\
\hline Europe & 2 & $1.02(1.00-1.05)$ & 0 & 0.59 \\
\hline North America & 2 & 1.07 (0.95-1.09) & 82.7 & 0.02 \\
\hline Asia & 1 & $1.00(0.97-1.04)$ & - & - \\
\hline Australia & 1 & $1.12(0.99-1.27)$ & - & - \\
\hline & & .1 unit & & \\
\hline & $\bar{n}$ & $\mathrm{RR}(95 \% \mathrm{CI})$ & $I^{2}(\%)$ & $P_{\mathrm{h}}$ \\
\hline Colorectal cancer & & & & \\
\hline All studies & 6 & $1.03(1.01-1.05)$ & 15.7 & 0.31 \\
\hline Stratified by sex & & & & \\
\hline Men & 2 & $1.18(0.88-1.60)$ & 81.8 & 0.02 \\
\hline Women & 4 & $1.03(1.01-1.04)$ & 0 & 0.72 \\
\hline Stratified by geographic lo & & & & \\
\hline Europe & - & - & - & - \\
\hline North America & 4 & $1.04(1.00-1.08)$ & 0 & 0.99 \\
\hline Asia & 2 & $1.02(0.98-1.06)$ & 68 & 0.08 \\
\hline Australia & - & - & - & - \\
\hline Duration of follow-up & & & & \\
\hline$<10$ years follow-up & 1 & $1.04(1.00-1.08)$ & - & - \\
\hline $10-<15$ years follow-up & 1 & $1.02(0.97-1.07)$ & - & - \\
\hline$\geq 15$ years follow-up & 4 & $1.06(0.98-1.14)$ & 52.5 & 0.12 \\
\hline Assessment of waist-to-hi & & & & \\
\hline Measured & 2 & $1.08(0.95-1.22)$ & 76.2 & 0.04 \\
\hline Self-reported & 2 & $1.02(0.99-1.04)$ & 36.7 & 0.21 \\
\hline Number of cases & & & & \\
\hline Cases $<1000$ & 5 & $1.04(1.00-1.08)$ & 30.5 & 0.23 \\
\hline Cases $1000-3000$ & 1 & $1.03(1.01-1.04)$ & - & - \\
\hline Cases $\geq 3000$ & - & - & - & - \\
\hline Colon cancer & & & & \\
\hline All studies & 7 & $1.16(1.05-1.28)$ & 82.5 & $<0.001$ \\
\hline Stratified by sex & & & & \\
\hline Men & 4 & $1.17(1.05-1.30)$ & 88.2 & $<0.001$ \\
\hline Women & 5 & $1.07(1.00-1.15)$ & 60.8 & 0.04 \\
\hline Stratified by geographic lo & & & & \\
\hline Europe & 1 & $1.27(1.13-1.43)$ & - & - \\
\hline North America & 3 & $1.14(0.98-1.32)$ & 78.5 & 0.01 \\
\hline Asia & 2 & $1.05(1.00-1.10)$ & 0 & 0.46 \\
\hline Australia & 1 & $1.47(1.27-1.71)$ & - & - \\
\hline
\end{tabular}


Table 1 (continued)

\begin{tabular}{|c|c|c|c|c|}
\hline & \multicolumn{4}{|c|}{ Waist-to-hip ratio, per 0.1 unit } \\
\hline & $n$ & $\mathrm{RR}(95 \% \mathrm{CI})$ & $I^{2}(\%)$ & $P_{\mathrm{h}}$ \\
\hline \multicolumn{5}{|c|}{ Proximal colon cancer } \\
\hline All studies & 4 & $1.13(1.00-1.28)$ & 72.5 & $<0.01$ \\
\hline \multicolumn{5}{|l|}{ Stratified by sex } \\
\hline Men & 2 & $1.41(1.17-1.72)$ & 0 & 0.68 \\
\hline Women & 3 & $1.03(0.96-1.11)$ & 43.2 & 0.17 \\
\hline \multicolumn{5}{|c|}{ Stratified by geographic location } \\
\hline Europe & - & - & - & - \\
\hline North America & 3 & $1.06(0.95-1.09)$ & 71.9 & 0.03 \\
\hline Asia & - & - & - & - \\
\hline Australia & 1 & $1.36(1.10-1.38)$ & 0 & 0.49 \\
\hline \multicolumn{5}{|l|}{ Distal colon cancer } \\
\hline All studies & 4 & $1.17(1.01-1.35)$ & 74.6 & $<0.01$ \\
\hline \multicolumn{5}{|l|}{ Stratified by sex } \\
\hline Men & 2 & $1.50(0.91-2.47)$ & 82.9 & 0.01 \\
\hline Women & 3 & $1.04(1.02-1.06)$ & 0 & 0.73 \\
\hline \multicolumn{5}{|c|}{ Stratified by geographic location } \\
\hline Europe & - & - & - & - \\
\hline North America & 3 & $1.04(1.02-1.06)$ & 0 & 0.58 \\
\hline Asia & - & - & - & - \\
\hline Australia & 1 & $1.52(0.92-1.50)$ & 78.5 & 0.03 \\
\hline \multicolumn{5}{|l|}{ Rectal cancer } \\
\hline All studies & 6 & $1.04(1.01-1.08)$ & 22.1 & 0.26 \\
\hline \multicolumn{5}{|l|}{ Stratified by sex } \\
\hline Men & 4 & $1.05(1.02-1.09)$ & 0 & 0.62 \\
\hline Women & 4 & $1.06(0.98-1.15)$ & 44.9 & 0.14 \\
\hline \multicolumn{5}{|c|}{ Stratified by geographic location } \\
\hline Europe & 1 & $1.04(0.98-1.09)$ & - & - \\
\hline North America & 2 & $1.03(0.98-1.09)$ & 0 & 0.63 \\
\hline Asia & 2 & $1.12(0.98-1.28)$ & 0 & 0.96 \\
\hline Australia & 1 & $1.24(1.02-1.51)$ & - & - \\
\hline
\end{tabular}

$R R$ relative risk; 95\% CI 95\% confidence interval

increased risk with increasing height (Supplemental Fig. 1B).

\section{Height and proximal and distal colon cancer}

Four studies were included in the dose-response metaanalysis of height and proximal (1326 cases) and distal (1275 cases) colon cancer.

The summary RR for proximal colon cancer per an increase of $5 \mathrm{~cm}$ was 1.02 (95\% CI 0.99-1.05) (Table 1 and Supplemental Fig. 2A). High heterogeneity was observed $\left(I^{2}=76 \%, P_{\text {heterogeneity }}<0.01\right)$. There was no evidence of publication bias $(P$ value Egger's test $=0.96)$.

In stratified analysis by sex, no significant association was observed. In stratified analysis by geographical location, a borderline significant increased risk was observed in studies conducted in North America.

There was no evidence of a non-linear association $\left(P_{\text {nonlinearity }}=0.41, n=3\right)$ (Supplemental Fig. 2B).

The summary RR for distal colon cancer per an increase of $5 \mathrm{~cm}$ was 1.01 (95\% CI 1.00-1.01) (Table 1; Supplemental Fig. 3A). No heterogeneity was observed $\left(I^{2}=0 \%\right.$, $\left.P_{\text {heterogeneity }}=0.85\right)$. There was a significant evidence of publication bias ( $P$ value Egger's test $=0.04)$.

In stratified analysis by sex and geographical location, positive associations were borderline significant in studies in women and not in men, and in studies conducted in North America.

There was an evidence of a significant non-linear association $\left(P_{\text {nonlinearity }}=0.01, n=3\right)($ Supplemental Fig. 3B $)$. 
A weight and colorectal cancer, per $5 \mathrm{~cm}$

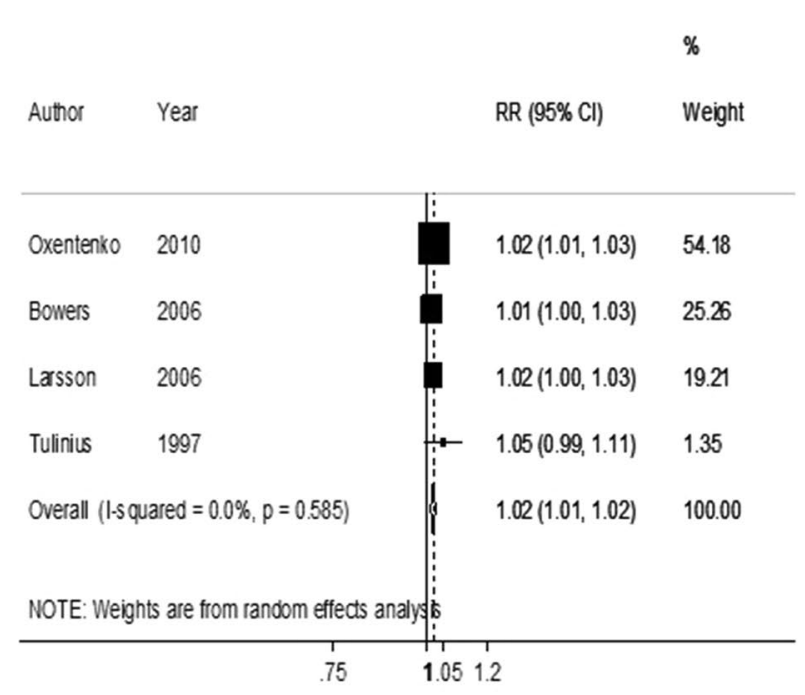

B weight and colorectal cancer, nonlinear dose-response

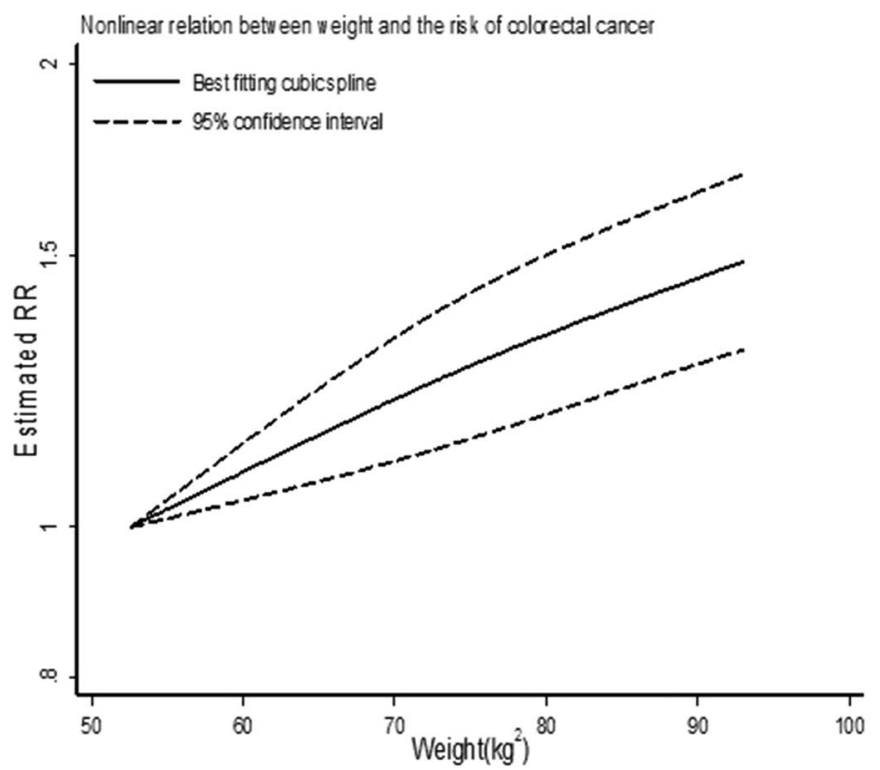

Fig. 3 Weight and colorectal cancer risk (dose-response and nonlinear analysis). RR relative risk, 95\% CI 95\% confidence interval. Summary RR calculated using a random-effects model

\section{Height and rectal cancer}

Fourteen studies $(30,762$ cases $)$ were included in the dose-response meta-analysis $[4,5,8,11,26,30,33,41,45$, $58,64-66]$. The summary RR for an increase of $5 \mathrm{~cm}$ was 1.01 (95\% CI 1.00-1.02) (Table 1; Supplemental Fig. 4A). There was high heterogeneity $\left(I^{2}=62 \%, P_{\text {heterogeneity }}=\right.$ $0.002)$. There was evidence of a significant publication or small study bias ( $P$ value Egger's test $<0.001$ ).

In stratified analysis, the summary RR showed a slightly stronger association in studies in men than in women and a stronger association in studies in North America than in studies in Asia and Europe (Table 1).

There was no evidence of a non-linear association $\left(P_{\text {nonlinearity }}=0.08, n=9\right)($ Supplemental Fig. 4B).

\section{Weight}

\section{Weight and colorectal cancer}

Four studies ( 2700 cases) were included in the meta-analysis $[9,26,27,39]$. The summary RR per an increase of $5 \mathrm{~kg}$ was 1.02 (95\% CI 1.01-1.02) (Fig. 3a) and there was no evidence.

of heterogeneity $\left(I^{2}=0 \%, P_{\text {heterogeneity }}=0.58\right)$. There was no evidence of a publication or small study bias ( $P$ value Egger's test $=0.49$ ).

In stratified analysis by sex and geographical location, positive significant associations were almost similar in both men and women and in studies conducted in Europe and North America.

There was no significant evidence of a non-linear association $\left(P_{\text {nonlinearity }}=0.43, n=3\right)$ (Fig. 3b).

\section{Weight and colon cancer}

Six studies ( 2143 cases) were included in the meta-analysis [26, 27, 45, 68-70]. The summary RR per an increase of $5 \mathrm{~kg}$ was 1.02 (95\% CI 1.01-1.03) (Table 1; Supplemental Fig. 5A). No heterogeneity was observed $\left(I^{2}=18 \%\right.$, $\left.P_{\text {heterogeneity }}=0.29\right)$. There was evidence of a significant publication or small study bias ( $P$ value Egger's test $=0.01$ ).

In stratified analysis, the summary RR showed stronger associations in studies in women than in men and in studies conducted in Australia than in Europe (Table 1).

There was no significant evidence of a non-linear association $\left(P_{\text {nonlinearity }}=0.84, n=3\right)($ Supplemental Fig. 5B).

\section{Weight and proximal and distal colon cancer}

Three studies were included in dose-response meta-analysis for proximal (1748 cases) and distal (1083 cases) colon cancer.

The summary RR for proximal colon cancer per an increase of $5 \mathrm{~kg}$ was 1.02 (95\% CI 1.00-1.04) (Table 1; Supplemental Fig. 6A). No heterogeneity was observed ( $I^{2}$ $\left.=34 \%, P_{\text {heterogeneity }}=0.22\right)$. There was no evidence of publication bias $(P$ value Egger's test $=0.73)$. 
A BMI and colorectal cancer, per $5 \mathrm{~kg} / \mathrm{m}^{2}$

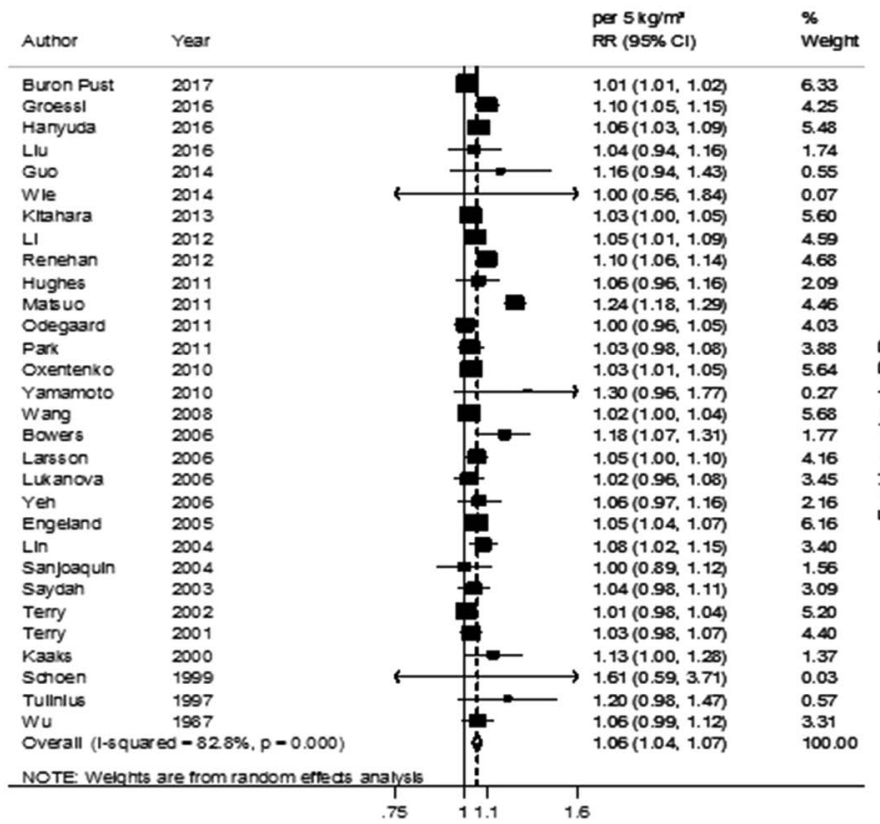

B BMI and colorectal cancer, nonlinear dose-response

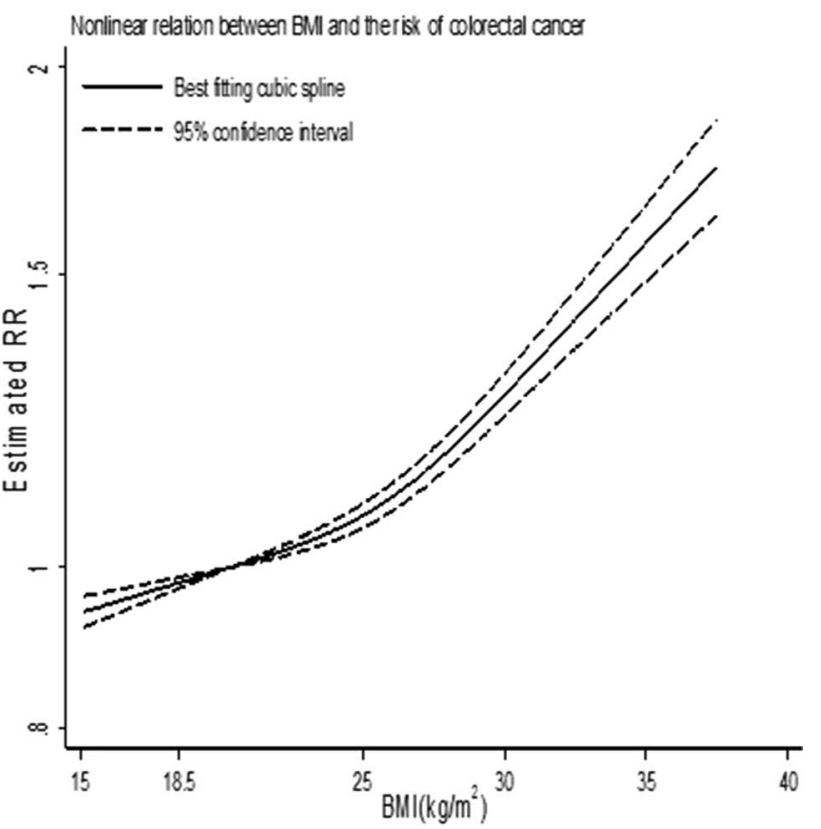

Fig. 4 BMI and colorectal cancer risk (dose-response and nonlinear analysis). RR relative risk, 95\% CI 95\% confidence interval. Summary RR calculated using a random-effects model

In stratified analysis by sex, no significant association was observed.

There was no significant evidence of a non-linear association $\left(P_{\text {nonlinearity }}=0.31, n=2\right)$ (Supplemental Fig. 6B).

The summary RR for distal colon cancer per an increase of $5 \mathrm{~cm}$ was 1.03 (95\% CI 1.01-1.05) (Table 1; Supplemental Fig. 7A). No heterogeneity was observed $\left(I^{2}=23 \%\right.$, $\left.P_{\text {heterogeneity }}=0.27\right)$. There was no evidence of publication bias $(P$ value Egger's test $=0.76)$.

In stratified analysis by sex, no significant association was observed.

There was no significant evidence of a non-linear association $\left(P_{\text {nonlinearity }}=0.48, n=2\right)($ Supplemental Fig. 7B $)$.

\section{Weight and rectal cancer}

Four studies (1186 cases) were included in the meta-analysis [26, 27, 41, 45]. The summary RR per an increase of $5 \mathrm{~kg}$ was 1.01 (95\% CI 1.00-1.02) (Table 1; Supplemental Fig. 8A). No heterogeneity was observed $\left(I^{2}=0 \%\right.$, $\left.P_{\text {heterogeneity }}=0.65\right)$ (Table 1$)$.

In stratified analysis by geographical location, positive significant association was observed in studies in Europe.

There was no significant evidence of a non-linear association $\left(P_{\text {nonlinearity }}=0.29, n=3\right)($ Supplemental Fig. $8 \mathrm{~B})$.

\section{Body mass index (BMI)}

\section{BMI and colorectal cancer}

Thirty-eight studies (84 859 cases) were included in the dose-response meta-analysis [8, 9, 11, 14, 15, 18-24, 26-32, $34-40,50,51]$. The summary RR for an increase of $5 \mathrm{~kg} / \mathrm{m}^{2}$ was 1.06 (95\% CI 1.04-1.07) (Fig. 4a). There was evidence of high heterogeneity $\left(I^{2}=83 \%, P_{\text {heterogeneity }}<0.001\right)$. There was evidence of a publication or small study bias ( $P$ value Egger's test $<0.001)$.

Several differences in associations emerged in stratified analyses by study size, years of follow-up and in studies in which weight and height were self-assessed and in those in which they were measured, but none of them were statistically significant (Table 1). The associations tended to be stronger in men than in women and when the analysis was restricted to studies that reported in both sex, the summary RR were 1.05 (95\% CI 1.03-1.07) in women and 1.08 (95\% CI 1.05-1.12) in men. The association was stronger in studies in Asia than in studies conducted in Europe and North America. The associations tended to be stronger in studies in which weight and height were self-reported compared to measured, in studies with higher number of cases ( $>3000$ ), and in studies with $10-<15$ years of follow-up. However, none of these variables independently explained the heterogeneity. 
There was statistical evidence of a non-linear relationship $\left(P_{\text {nonlinearity }}<0.001, n=29\right)$ (Fig. $\left.4 b\right)$. Colorectal cancer risk increased with increasing BMI and the risk appeared to be stronger from a BMI increases around $27 \mathrm{~kg} / \mathrm{m}^{2}$ and above.

\section{BMI and colon cancer}

Forty two studies $(85,295$ cases) were included in the dose-response meta-analysis [11, 14, 18-21, 24, 26-28, 30, $31,33,35-37,44,45,49,66,69-83]$. The summary RR for an increase of $5 \mathrm{~kg} / \mathrm{m}^{2}$ was 1.07 (95\% CI 1.05-1.08) (Table 1; Supplemental Fig. 9A). There was high heterogeneity $\left(I^{2}=79 \%, P_{\text {heterogeneity }}<0.001\right)$. There was evidence of publication or small study bias ( $P$ value Egger's test $<0.001)$.

In analysis stratified by sex and geographical location, the summary RR showed stronger associations in studies in men than women and stronger associations in studies in Asia and North America than studies in Europe (Table 1).

There was no statistical evidence of a non-linear association $\left(P_{\text {nonlinearity }}=0.09, n=33\right)$ (Supplemental Fig. 9B).

\section{BMI and proximal and distal colon cancer}

Twenty studies were included in dose-response meta-analysis for proximal (8437 cases) and distal (14,985 cases) colon cancer. There was evidence of significant positive association between BMI and both proximal and distal colon cancer risk, that was slightly stronger for distal than for proximal cancer.

The summary RR for proximal colon cancer per an increase of $5 \mathrm{~kg} / \mathrm{m}^{2}$ was 1.05 (95\% CI 1.03-1.08) (Table 1;
Supplemental Fig. 10A). Medium heterogeneity was observed $\left(I^{2}=44 \%, P_{\text {heterogeneity }}=0.04\right)$. There was no evidence of publication bias ( $P$ value Egger's test $=0.06)$.

In stratified analysis by sex and geographical location, positive significant associations were stronger in men than in women and in studies conducted in Asia than in North America.

There was no statistical evidence of a non-linear association $\left(P_{\text {nonlinearity }}=0.14, n=8\right)$ (Supplemental Fig. 10B).

The summary RR for distal colon cancer for an increase of $5 \mathrm{~kg} / \mathrm{m}^{2}$ was 1.08 (95\% CI 1.04-1.11) (Table 1; Supplemental Fig. 11A). High heterogeneity was observed $\left(I^{2}\right.$ $=52 \%, P_{\text {heterogeneity }}=0.02$ ). There was no evidence of publication bias ( $P$ value Egger's test $=0.08$ ).

In stratified analysis by sex and geographical location, positive significant associations were stronger in men than in women and in studies conducted in Asia than in Europe and North America.

There was no statistical evidence of a non-linear association $\left(P_{\text {nonlinearity }}=0.49, n=8\right)$ (Supplemental Fig. 11B).

\section{BMI and rectal cancer}

Thirty-six studies $(73,186$ cases $)$ were included in the dose-response meta-analysis [8, 11, 14, 17-21, 24, 26-28, $30,31,33,35,36,41,45,49,66,70-72,75,76,79,80]$. The summary RR for an increase of $5 \mathrm{~kg} / \mathrm{m}^{2}$ was 1.02 (95\% CI 1.01-1.03) (Table 1; Supplemental Fig. 12A). There was no evidence of heterogeneity $\left(I^{2}=29.3 \%\right.$, $\left.P_{\text {heterogeneity }}=0.07\right)$. No evidence of publication or small study bias was detected ( $P$ value Egger's test $=0.08)$.
A Waist circumference and colorectal cancer, per $10 \mathrm{~cm}$

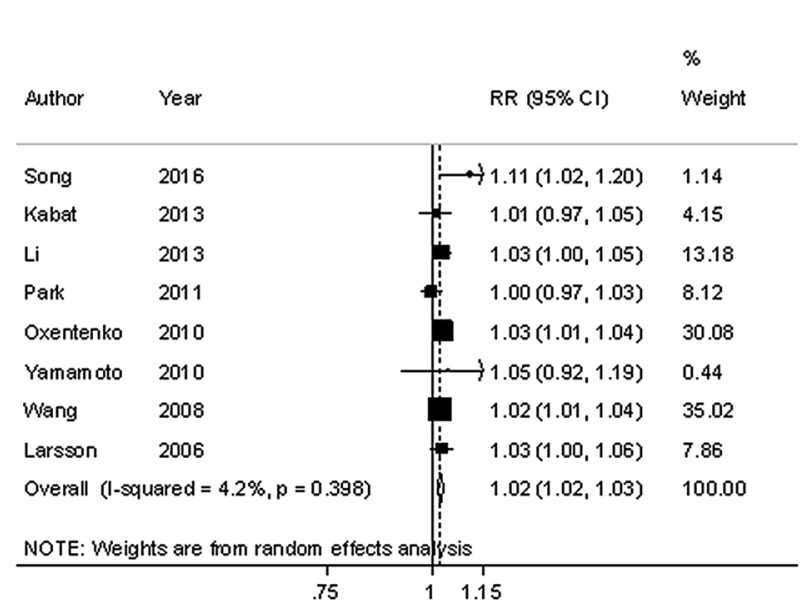

B Waist circumference and colorectal cancer, nonlinear dose-response

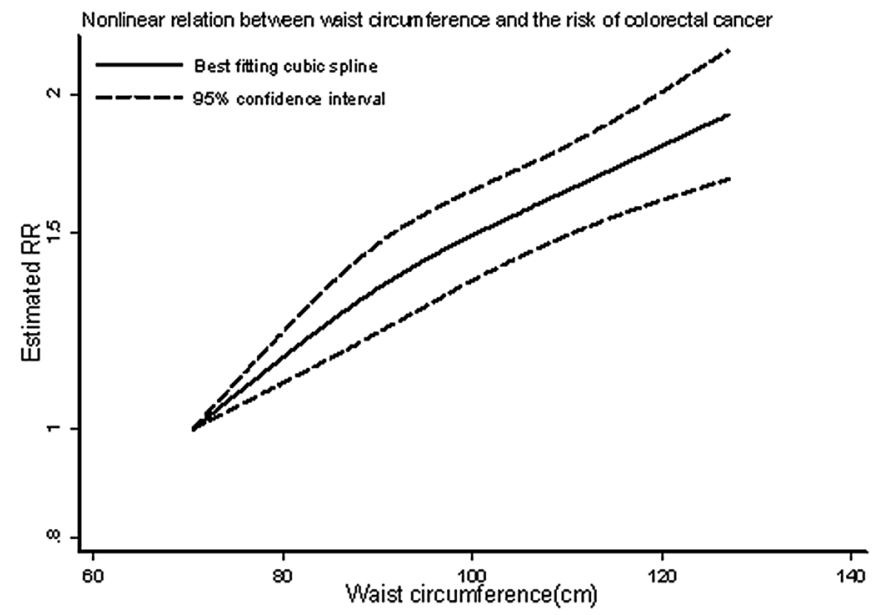

Fig. 5 Waist circumference and colorectal cancer risk (dose-response analysis). $R R$ relative risk, 95\% CI 95\% confidence interval. Summary RR calculated using a random-effects model 
In stratified analysis, the summary RR was statistically significant in men but not in women, and in studies in Asia and not in other geographic locations (Table 1).

There was evidence of a non-linear association $\left(P_{\text {nonlinearity }}<0.001, n=28\right)$ (Supplemental Fig. 12B). The curve shows that there is no evidence of association for $\mathrm{BMI}<27.5 \mathrm{~kg} / \mathrm{m}^{2}$, but increased risk for BMI values above this level.

\section{Waist circumference}

\section{Waist circumference and colorectal cancer}

Ten studies ( 1884 cases) were included in the dose-response meta-analysis [9, 16, 18, 22-24, 27, 53]. The summary RR for an increase of $10 \mathrm{~cm}$ was 1.02 (95\% CI 1.02-1.03) (Fig. 5a). There was no evidence of heterogeneity $\left(I^{2}=4 \%\right.$, $\left.P_{\text {heterogeneity }}=0.40\right)$ (Fig. 4a). There was no evidence of publication bias $(P$ value Egger's test $=0.60)$.

In stratified analysis, the positive associations were significant only in women and not in men, in studies conducted in Asia and North America and not in studies in Europe, and in studies with less years of follow-up ( $<10$ years). In studies with measured or self-reported waist circumferences, the positive association was similar (Table 1).

There was no evidence of non-linear association $\left(P_{\text {nonlinearity }}=0.17, n=7\right)$ (Fig. $\left.5 b\right)$.

\section{Waist circumference and colon cancer}

Eleven studies (4729 cases) were included in the dose-response meta-analysis [16, 18, 24, 27, 42-45, 53, 68]. The summary RR for an increase of $10 \mathrm{~cm}$ was 1.05 (95\% CI 1.02-1.07) (Table 1; Supplemental Fig. 13A). High heterogeneity was observed $\left(I^{2}=72 \%, P_{\text {heterogeneity }}=<0.001\right)$. There was evidence of publication bias ( $P$ value Egger's test $<0.01$ ).

In stratified analysis by sex and geographic location, the positive significant associations were stronger in men than in women and in studies in Asia and North America compared to the studies conducted in Europe (Table 1).

There was a significant evidence of non-linear association $\left(P_{\text {nonlinearity }}=0.001, n=8\right)$. The curve shows increased risk of colon cancer with increasing waist circumference, with steeper associations at lower levels compared to higher levels of waist circumference (Supplemental Fig. 13B).

\section{Waist circumference and proximal and distal colon cancer}

Five studies were included in dose-response meta-analysis for proximal (1982 cases) and distal (1320 cases) colon cancer.
There were significant positive associations of similar magnitude between waist circumference and both proximal and distal colon cancer.

The summary RR for proximal colon cancer for an increase of $10 \mathrm{~cm}$ was 1.05 (95\% CI 1.01-1.09). No heterogeneity was observed $\left(I^{2}=49.9 \%, P_{\text {heterogeneity }}=0.09\right)$ (Table 1; Supplemental Fig. 14A). There was no evidence of publication bias ( $P$ value Egger's test $=0.41)$.

In stratified analysis by sex and geographical location, positive significant associations were observed in studies in women and not in men, and in studies conducted in North America.

There was no statistical evidence of a non-linear association $\left(P_{\text {nonlinearity }}=0.41, n=3\right)$ (Supplemental Fig. 14B).

The summary RR for distal colon cancer per an increase of $10 \mathrm{~cm}$ was 1.06 (95\% CI 1.02-1.11). No heterogeneity was observed $\left(R=50.5 \%, P_{\text {heterogeneity }}=0.09\right)($ Table 1 ; Supplemental Fig. 15A). There was no evidence of publication bias $(P$ value Egger's test $=0.17)$.

In stratified analysis by sex and geographical location, positive significant associations were observed in studies in women and not in men, and in studies conducted in North America.

There was no statistical evidence of a non-linear association $\left(P_{\text {nonlinearity }}=0.84, n=3\right)$ (Supplemental Fig. 15B).

\section{Waist circumference and rectal cancer}

Eight studies (1980 cases) were included in the dose-response meta-analysis of waist circumference and rectal cancer [18, 24, 27, 41, 45, 53]. The summary RR for an increase of $10 \mathrm{~cm}$ was 1.03 (95\% CI 1.00-1.05) (Table 1; Supplemental Fig. 16A). No heterogeneity was observed ( $I^{2}$

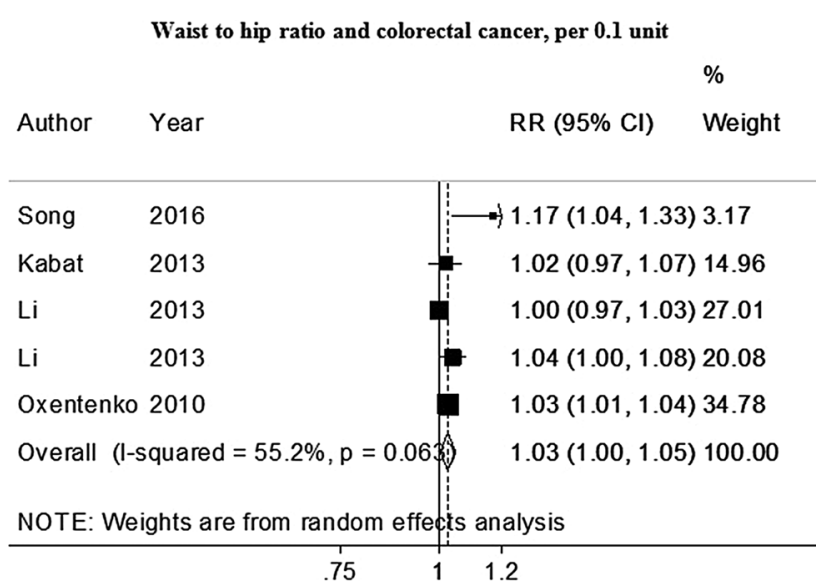

Fig. 6 Waist-to-hip ratio and colorectal cancer risk (dose-response and nonlinear analysis). $R R$ relative risk, 95\% CI 95\% confidence interval. Summary RR calculated using a random-effects model 
$\left.=46 \%, P_{\text {heterogeneity }}=0.10\right)$. There was no evidence of publication bias $(P$ value Egger's test $=0.30)$.

In stratified analysis by sex, significant association was observed only in women and not in men. In stratified analysis by geographic location, the positive association was borderline significant in studies in Europe and not significant in studies conducted in North America (Table 1).

There was no evidence of non-linear association $\left(P_{\text {nonlinearity }}=0.40, n=6\right)($ Supplemental Fig. 16B).

\section{Waist-to-hip ratio}

\section{Waist-to-hip ratio and colorectal cancer}

Six studies (4 689 cases) were included in the dose-response meta-analysis of waist to hip ratio and colorectal cancer [9, $16,18,49,53]$. The summary RR for an increase of 0.1 unit was 1.03 (95\% CI 1.01-1.05) (Fig. 6).

No heterogeneity was observed $\left(I^{2}=16 \%, P_{\text {heterogeneity }}\right.$ $=0.31)$. There was no evidence of publication bias $(P$ value Egger's test $=0.436$ ).

In stratified analysis, positive significant associations were observed only in studies in women and not in men, and in studies in North America and not in studies conducted in Asia (Table 1).

There was no statistical evidence of a non-linear association $\left(P_{\text {nonlinearity }}=0.34, n=4\right)$ (Supplemental Fig. 6B).

\section{Waist-to-hip ratio and colon cancer}

Seven studies (3126 cases) were included in the dose-response meta-analysis of waist to hip ratio and colon cancer $[18,42,43,45,49,53,68,84]$. The summary RR for an increase of 0.1 unit was 1.16 (95\% CI 1.05-1.28) (Table 1; Supplemental Fig. 17A).

High heterogeneity was observed $\left(I^{2}=82 \%\right.$, $\left.P_{\text {heterogeneity }}<0.001\right)$. There was no evidence of publication bias $(P$ value Egger's test $=0.14)$.

In stratified analysis by sex and geographic location, stronger association was observed in men compared to women and in studies in Europe than in studies in Asia (Table 1).

There was a significant evidence of non-linear association, showing colon cancer risk increased with increasing waist-to-hip-ratio $\left(P_{\text {nonlinearity }}=0.001, n=7\right)$ and the curve was approximately linear across the range of waist-to-hip ratio values (Supplemental Fig. 17B).

\section{Waist-to-hip ratio and proximal and distal colon cancer}

Four studies were included in dose-response meta-analysis for proximal (1073 cases) and distal (932 cases) colon cancer. The summary RR for proximal colon cancer for an increase of 0.1 unit was $1.13(1.00-1.28)$. High heterogeneity was observed $\left(I^{2}=72.5 \%, P_{\text {heterogeneity }}<0.01\right)$ (Table 1 ; Supplemental Fig. 18). There was no evidence of publication bias ( $P$ value Egger's test $=0.62$ ).

In stratified analysis by sex, the positive association was only significant in men and not in women.

There was not enough studies to conduct non-linear analysis $(n=1)$.

The summary RR for distal colon cancer for an increase of 0.1 unit was 1.17 (95\% CI 1.01-1.35). High heterogeneity was observed $\left(I^{2}=74.6 \%, P_{\text {heterogeneity }}<0.01\right)$ (Table 1 and Supplemental Fig. 19). There was no evidence of publication bias ( $P$ value Egger's test $=0.28$ ).

In stratified analysis by sex and geographical location, the positive associations were significant only in women and not in men, and in studies in North America.

There was not enough studies to conduct non-linear analysis $(n=1)$.

\section{Waist-to-hip ratio and rectal cancer}

Six studies (1510cases) were included in the dose-response meta-analysis of waist to hip ratio and rectal cancer risk $[18,41,45,49,53]$. The summary RR for an increase of 0.1 unit was 1.04 (95\% CI 1.01-1.08) (Table 1; Supplemental Fig. 20A). No heterogeneity was observed $\left(I^{2}=22 \%\right.$, $\left.P_{\text {heterogeneity }}=0.26\right)$. There was no evidence of publication bias $(P$ value Egger's test $=0.42)$.

In stratified analysis by sex, positive association was only significant in men and not in women. In stratified analysis by location, no significant association was observed (Table 1).

There was no statistical evidence of a non-linear association $\left(P_{\text {nonlinearity }}=0.14, n=3\right)$ (Supplemental Fig. 20B).

\section{Discussion}

In this meta-analysis of prospective studies, we quantified the evidence for the association between adult height, general obesity and abdominal fatness with CRC risk. To our knowledge this is the first dose-response meta-analysis to investigate the association between adult height and CRC risk. We found evidence of an increased risk of colorectal cancer with greater adult height. The association shows a $4 \%$ increased risk per an increment of $5 \mathrm{~cm}$ of height for colorectal, 2 and 1\% increased risk for colon cancer and rectal cancer, respectively. The trend of increasing risk with greater adult height was similar in men and women, which is in contradiction with the results of several previous observational studies, showing stronger association among women than in men $[8,9,12,26,30,45,65,67,68]$. Also stronger association was observed in studies in which height was measured rather than self-reported. Moreover, there was an 
evidence of a non-linear association for greater adult height and colon cancer risk, with steeper associations at lower compared to higher levels of height.

The specific mechanism explaining the association between greater height and colorectal cancer risk has not been elucidated, although there is a great deal of evidences for an association between greater height and increased risk of other cancers including breast, pancreas, endometrium and ovarian cancers $[85,86]$. Taller people have a greater number of cells in their body [87] and it has also been suggested that height is associated with the length of the intestines [88]; therefore, taller people might have a higher risk of cell mutations leading to malignancy. Elevated levels of insulin-like growth factor-1 (IGF-1) may play an important role in determining growth as higher IGF-1 levels in childhood are associated with childhood growth [34]. Elevated levels of IGF-1 may contribute to cancer risk by inhibiting apoptosis, stimulating cell proliferation and synthesis of sex steroids and inhibiting the synthesis of steroid hormone binding globulin [85, 89]. Nutritional status and diseases particularly infections during childhood and adolescence are play an important role in determining adult height $[85,90$, 91] as poor nutrition and infections are the main reasons of growth failures in early childhood [92, 93]. Furthermore, it has been suggested that adult height represents the balance between nutritional intake and losses over time, specifically during the growth periods, including losses due to physical activity, psychological stress, and disease from the conception to maturity [92] and consequently adult height is a product of cumulative net nutrition [92]. Cancer risk in adulthood might be related to early life conditions but why these conditions might differentially affect men and women is unknown.

Our findings also show a positive relationship between all the included anthropometric factors (weight, BMI, waist circumference and waist to hip ratio) and the risk of colorectal cancer, and all the anatomical localizations (colon, proximal colon, distal colon, and rectal). Almost all positive associations were statistically significant.

The strongest association for BMI was observed with distal colon cancer, showing $8 \%$ increased risk for an increase of $5 \mathrm{~kg} / \mathrm{m}^{2}$ of BMI. The trend of increasing colorectal cancer risk with greater BMI was stronger in men than in women.

For waist circumference and waist to hip ratio, the strongest associations were observed with colon cancer, showing 5 and $16 \%$ increased risk per an increase of $10 \mathrm{~cm}$ of waist circumference and 0.1 increment of waist to hip ratio, respectively. Our results for an association between BMI and waist circumference and colorectal cancer risk are in agreement with the findings of two previous metaanalyses $[47,48]$.

We observed a nonlinear association between BMI and colorectal cancer risk. Colorectal cancer risk increased with
BMI which appears to be stronger from BMI above $27 \mathrm{~kg} /$ $\mathrm{m}^{2}$ approximately. There was also evidence of non-linear association $(P=0.02)$ for BMI and colon cancer risk but the curve appeared approximately linear across the range of BMI values investigated. Furthermore, nonlinear associations were observed for abdominal fatness measures (waist circumference and waist to hip ratio) and colon cancer risk, with steeper associations at lower levels compared to higher levels of waist circumference. Several mechanisms are hypothesized to link obesity to colorectal cancer. Metabolic syndrome, elevated levels of insulin and insulin-like growth factors which inhibits apoptosis and modulates cell proliferation are suggested to play a role in the aetiology of colorectal cancer [48, 94-96]. There are evidences suggesting that abdominal fatness measured by high waist circumference and waist to hip ratio is a better indicator of metabolic disturbances, that affect the risk of colorectal cancer, than general obesity measured by high BMI as BMI has the limitation of not distinguishing between fat mass and lean mass [53].

This study has several advantages. It is based on largescale prospective studies which minimize the probability of recall or selection bias, and also includes a large number of studies with relatively long follow-up and large number of cases that significantly increase the statistical power of the analysis. Moreover, the majority of the included studies in our meta-analysis were adjusted at least for age and other potential confounders such as alcohol consumption, smoking and physical activity.

However, the current meta-analysis has some limitations which should be taken into account when interpreting the results. High heterogeneity was observed across studies, which would affect the reliability of the summary RR estimates and lead to less accurate results. We did not find an explanation for this heterogeneity as it persisted in most subgroup analyses. This high heterogeneity might be due to variations and differences in anthropometric measurements categories. Another limitation of this meta-analysis can be due to measurement errors in the assessment of anthropometric measures, although most of the studies reported measured BMI $(n=27)$ rather than self-reported $(n=16)$.

In conclusion, our findings support the existing evidence of a positive association of general and abdominal body fatness with risk of CRC. In addition, higher adult height is significantly associated with increased colorectal cancer risk, particularly in women. These findings suggest that early life nutrition might play a role in colorectal cancer risk in adulthood.

Acknowledgements The authors' responsibilities were as followsL. A., A. R. V., J.G.S: performed the updated literature search and the updated data extraction; L. A.: conducted statistical analyses, wrote the first draft of the original manuscript, had primary responsibility for the final content of the manuscript, and took responsibility for the integrity of data and accuracy of the data analysis; C. S.: was database 
manager for the project; and all authors, D. A., D. C., S.S., S. V. and E.P., contributed to the revision of the manuscript and had full access to all data in the study. D. C. G.: advised on and contributed to statistical analyses. T. N. is the principal investigator of the Continuous Update Project at Imperial College. All authors commented on drafts of the paper and approved the final version. The views expressed in this review are the opinions of the authors. The views may not represent the views of World Cancer Research Fund International/American Institute for Cancer Research and may differ from those in future updates of the evidence related to food, nutrition, physical activity, and cancer risk. The sponsor of this study had no role in the decisions about the analysis or interpretation of the data; or preparation, review, or approval of the manuscript.

\section{Compliance with ethical standards}

Funding This work was funded by the World Cancer Research Fund network (grant number 2007/SP01) as part of the Continuous Update Project. The work of SS and DA was supported by a NHS BRC grant (Interventional Public Health).

Conflict of interest None of the authors reported a conflict of interest related to the study.

Open Access This article is distributed under the terms of the Creative Commons Attribution 4.0 International License (http://creativecommons.org/licenses/by/4.0/), which permits unrestricted use, distribution, and reproduction in any medium, provided you give appropriate credit to the original author(s) and the source, provide a link to the Creative Commons license, and indicate if changes were made.

\section{References}

1. Ferlay J, Soerjomataram I, Dikshit R et al (2015) Cancer incidence and mortality worldwide: sources, methods and major patterns in GLOBOCAN 2012. Int J Cancer 136:E359-E386

2. World Cancer Research Fund/American Institute forCancer Research (2011) Food, nutrition, physical activity, and the prevention of colorectal cancer. AICR, Washington, DC

\section{Ref Type: Online Source}

3. Boursi B, Haynes K, Mamtani R et al (2014) Height as an independent anthropomorphic risk factor for colorectal cancer. Eur J Gastroenterol Hepatol 26:1422-1427

4. Kabat GC, Kim MY, Hollenbeck AR et al (2014) Attained height, sex, and risk of cancer at different anatomic sites in the NIH-AARP diet and health study. Cancer Causes Control 25:1697-1706

5. Kabat GC, Heo M, Kamensky V et al (2013) Adult height in relation to risk of cancer in a cohort of Canadian women. Int J Cancer 132:1125-1132

6. Walter RB, Brasky TM, Buckley SA et al (2013) Height as an explanatory factor for sex differences in human cancer. J Natl Cancer Inst 105:860-868

7. Green J, Cairns BJ, Casabonne D et al (2011) Height and cancer incidence in the Million Women Study: prospective cohort, and meta-analysis of prospective studies of height and total cancer risk. Lancet Oncol 12:785-794
8. Hughes LA, Simons CC, van den Brandt PA et al (2011) Body size and colorectal cancer risk after 16.3 years of follow-up: an analysis from The Netherlands Cohort Study. Am J Epidemiol 174:1127-1139

9. Oxentenko AS, Bardia A, Vierkant RA et al (2010) Body size and incident colorectal cancer: a prospective study of older women. Cancer Prev Res (Phila) 3:1608-1620

10. Shin A, Joo J, Yang HR et al (2014) Risk prediction model for colorectal cancer: National Health Insurance Corporation study, Korea. PLoS One 9:e88079

11. Buron PA, Alison R, Blanks R et al (2017) Heterogeneity of colorectal cancer risk by tumour characteristics: large prospective study of UK women. Int J Cancer 140:1082-1090

12. Thrift AP, Gong J, Peters U et al (2015) Mendelian randomization study of height and risk of colorectal cancer. Int J Epidemiol 44:662-672

13. Khankari NK, Shu XO, Wen W et al (2016) Association between adult height and risk of colorectal, lung, and prostate cancer: results from meta-analyses of prospective studies and mendelian randomization analyses. PLoS Med 13:e1002118

14. Guo L, Li N, Wang G et al (2014) Body mass index and cancer incidence: a prospective cohort study in northern China. Zhonghua Liu Xing Bing Xue Za Zhi 35:231-236

15. Wie GA, Cho YA, Kang HH et al (2014) Red meat consumption is associated with an increased overall cancer risk: a prospective cohort study in Korea. Br J Nutr 112:238-247

16. Kabat GC, Heo M, Wactawski-Wende J et al (2013) Body fat and risk of colorectal cancer among postmenopausal women. Cancer Causes Control 24:1197-1205

17. Kitahara CM, Berndt SI, de Gonzalez AB et al (2013) Prospective investigation of body mass index, colorectal adenoma, and colorectal cancer in the prostate, lung, colorectal, and ovarian cancer screening trial. J Clin Oncol 31:2450-2459

18. Li H, Yang G, Xiang YB et al (2013) Body weight, fat distribution and colorectal cancer risk: a report from cohort studies of 134255 Chinese men and women. Int J Obes (Lond) 37:783-789

19. Renehan AG, Flood A, Adams KF et al (2012) Body mass index at different adult ages, weight change, and colorectal cancer risk in the National Institutes of Health-AARP Cohort. Am J Epidemiol 176:1130-1140

20. Matsuo K, Mizoue T, Tanaka K et al (2012) Association between body mass index and the colorectal cancer risk in Japan: pooled analysis of population-based cohort studies in Japan. Ann Oncol 23:479-90

21. Odegaard AO, Koh WP, Yu MC et al (2011) Body mass index and risk of colorectal cancer in Chinese Singaporeans: the Singapore Chinese Health Study. Cancer 117:3841-3849

22. Park JY, Mitrou PN, Keogh RH et al (2012) Self-reported and measured anthropometric data and risk of colorectal cancer in the EPIC-Norfolk study. Int J Obes (Lond) 36:107-18

23. Yamamoto S, Nakagawa T, Matsushita Y et al (2010) Visceral fat area and markers of insulin resistance in relation to colorectal neoplasia. Diabetes Care 33:184-189

24. Wang Y, Jacobs EJ, Patel AV et al (2008) A prospective study of waist circumference and body mass index in relation to colorectal cancer incidence. Cancer Causes Control 19:783-792

25. Reeves GK, Pirie K, Beral V et al (2007) Cancer incidence and mortality in relation to body mass index in the Million Women Study: cohort study. BMJ 335:1134

26. Bowers K, Albanes D, Limburg P et al (2006) A prospective study of anthropometric and clinical measurements associated with insulin resistance syndrome and colorectal cancer in male smokers. Am J Epidemiol 164:652-664

27. Larsson SC, Rutegard J, Bergkvist L et al (2006) Physical activity, obesity, and risk of colon and rectal cancer in a cohort of Swedish men. Eur J Cancer 42:2590-2597 
28. Lukanova A, Bjor O, Kaaks R et al (2006) Body mass index and cancer: results from the Northern Sweden Health and Disease Cohort. Int J Cancer 118:458-466

29. Yeh CC, You SL, Chen CJ et al (2006) Peanut consumption and reduced risk of colorectal cancer in women: a prospective study in Taiwan. World J Gastroenterol 12:222-227

30. Engeland A, Tretli S, Austad G et al (2005) Height and body mass index in relation to colorectal and gallbladder cancer in two million Norwegian men and women. Cancer Causes Control 16:987-996

31. Lin J, Zhang SM, Cook NR et al (2004) Body mass index and risk of colorectal cancer in women (United States). Cancer Causes Control 15:581-589

32. Sanjoaquin MA, Appleby PN, Thorogood M et al (2004) Nutrition, lifestyle and colorectal cancer incidence: a prospective investigation of 10998 vegetarians and non-vegetarians in the United Kingdom. Br J Cancer 90:118-121

33. Wei EK, Giovannucci E, Wu K et al (2004) Comparison of risk factors for colon and rectal cancer. Int J Cancer 108:433-442

34. Saydah SH, Platz EA, Rifai N et al (2003) Association of markers of insulin and glucose control with subsequent colorectal cancer risk. Cancer Epidemiol Biomark Prev 12:412-418

35. Terry PD, Miller AB, Rohan TE (2002) Obesity and colorectal cancer risk in women. Gut 51:191-194

36. Terry P, Giovannucci E, Bergkvist L et al (2001) Body weight and colorectal cancer risk in a cohort of Swedish women: relation varies by age and cancer site. Br J Cancer 85:346-349

37. Kaaks R, Toniolo P, Akhmedkhanov A et al (2000) Serum C-peptide, insulin-like growth factor (IGF)-I, IGF-binding proteins, and colorectal cancer risk in women. J Natl Cancer Inst 92:1592-1600

38. Schoen RE, Tangen CM, Kuller LH et al (1999) Increased blood glucose and insulin, body size, and incident colorectal cancer. J Natl Cancer Inst 91:1147-1154

39. Tulinius H, Sigfusson N, Sigvaldason H et al (1997) Risk factors for malignant diseases: a cohort study on a population of 22,946 Icelanders. Cancer Epidemiol Biomark Prev 6:863-873

40. Wu AH, Paganini-Hill A, Ross RK et al (1987) Alcohol, physical activity and other risk factors for colorectal cancer: a prospective study. Br J Cancer 55:687-694

41. MacInnis RJ, English DR, Haydon AM et al (2006) Body size and composition and risk of rectal cancer (Australia). Cancer Causes Control 17:1291-1297

42. Giovannucci E, Ascherio A, Rimm EB et al (1995) Physical activity, obesity, and risk for colon cancer and adenoma in men. Ann Intern Med 122:327-334

43. Folsom AR, Kushi LH, Anderson KE et al (2000) Associations of general and abdominal obesity with multiple health outcomes in older women: the Iowa Women's Health Study. Arch Intern Med 160:2117-2128

44. Moore LL, Bradlee ML, Singer MR et al (2004) BMI and waist circumference as predictors of lifetime colon cancer risk in Framingham Study adults. Int J Obes Relat Metab Disord 28:559-67

45. Pischon T, Lahmann PH, Boeing $\mathrm{H}$ et al (2006) Body size and risk of colon and rectal cancer in the European Prospective Investigation Into Cancer and Nutrition (EPIC). J Natl Cancer Inst 98:920-931

46. Gunter MJ, Hoover DR, Yu H et al (2008) Insulin, insulin-like growth factor-I, endogenous estradiol, and risk of colorectal cancer in postmenopausal women. Cancer Res 68:329-337

47. Ma Y, Yang Y, Wang F et al (2013) Obesity and risk of colorectal cancer: a systematic review of prospective studies. PLoS One 8:e53916

48. Robsahm TE, Aagnes B, Hjartaker A et al (2013) Body mass index, physical activity, and colorectal cancer by anatomical subsites: a systematic review and meta-analysis of cohort studies. Eur J Cancer Prev 22:492-505

49. Liu Y, Warren AS, Wen W et al (2016) Prospective cohort study of general and central obesity, weight change trajectory and risk of major cancers among Chinese women. Int J Cancer 139:1461-1470

50. Hanyuda A, Ogino S, Qian ZR et al (2016) Body mass index and risk of colorectal cancer according to tumor lymphocytic infiltrate. Int J Cancer 139:854-868

51. Groessl EJ, Allison MA, Larson JC et al (2016) Coffee consumption and the incidence of colorectal cancer in women. J Cancer Epidemiol 2016:6918431

52. Kabat GC, Kim MY, Peters U et al (2012) A longitudinal study of the metabolic syndrome and risk of colorectal cancer in postmenopausal women. Eur J Cancer Prev 21:326-332

53. Song M, Hu FB, Spiegelman D et al (2016) Long-term status and change of body fat distribution, and risk of colorectal cancer: a prospective cohort study. Int J Epidemiol 45:871-883

54. DerSimonian R, Laird N (1986) Meta-analysis in clinical trials. Control Clin Trials 7:177-188

55. Higgins JP, Thompson SG (2002) Quantifying heterogeneity in a meta-analysis. Stat Med 21:1539-1558

56. Hamling J, Lee P, Weitkunat R et al (2008) Facilitating metaanalyses by deriving relative effect and precision estimates for alternative comparisons from a set of estimates presented by exposure level or disease category. Stat Med 27:954-970

57. Egger M, Davey SG, Schneider M et al (1997) Bias in meta-analysis detected by a simple, graphical test. BMJ 315:629-634

58. Kabat GC, Anderson ML, Heo M et al (2013) Adult stature and risk of cancer at different anatomic sites in a cohort of postmenopausal women. Cancer Epidemiol Biomark Prev 22:1353-1363

59. Otani T, Iwasaki M, Inoue M (2005) Body mass index, body height, and subsequent risk of colorectal cancer in middle-aged and elderly Japanese men and women: Japan public health centerbased prospective study. Cancer Causes Control 16:839-750

60. Gunnell D, May M, Ben-Shlomo Y et al (2003) Height, leg length, and cancer: the Caerphilly Study. Nutr Cancer 47:34-39

61. Hebert PR, Ajani U, Cook NR et al (1997) Adult height and incidence of cancer in male physicians (United States). Cancer Causes Control 8:591-597

62. Kato I, Akhmedkhanov A, Koenig K et al (1997) Prospective study of diet and female colorectal cancer: the New York University Women's Health Study. Nutr Cancer 28:276-281

63. Albanes D, Jones DY, Schatzkin A et al (1988) Adult stature and risk of cancer. Cancer Res 48:1658-1662

64. Sung J, Song YM, Lawlor DA et al (2009) Height and site-specific cancer risk: a cohort study of a korean adult population. Am J Epidemiol 170:53-64

65. Shimizu N, Nagata C, Shimizu H et al (2003) Height, weight, and alcohol consumption in relation to the risk of colorectal cancer in Japan: a prospective study. Br J Cancer 88:1038-1043

66. Thune I, Lund E (1996) Physical activity and risk of colorectal cancer in men and women. Br J Cancer 73:1134-1140

67. Bostick RM, Potter JD, Kushi LH et al (1994) Sugar, meat, and fat intake, and non-dietary risk factors for colon cancer incidence in Iowa women (United States). Cancer Causes Control 5:38-52

68. MacInnis RJ, English DR, Hopper JL et al (2006) Body size and composition and colon cancer risk in women. Int $\mathrm{J}$ Cancer 118:1496-1500

69. Bassett JK, Severi G, English DR et al (2010) Body size, weight change, and risk of colon cancer. Cancer Epidemiol Biomark Prev 19:2978-2986

70. Chyou PH, Nomura AM, Stemmermann GN (1996) A prospective study of colon and rectal cancer among Hawaii Japanese men. Ann Epidemiol 6:276-182 
71. Bhaskaran K, Douglas I, Forbes H et al (2014) Body-mass index and risk of 22 specific cancers: a population-based cohort study of 5.24 million UK adults. Lancet 384:755-765

72. Song X, Pukkala E, Dyba T et al (2014) Body mass index and cancer incidence: the FINRISK study. Eur J Epidemiol 29:477-487

73. Poynter JN, Inoue-Choi M, Ross JA et al (2013) Reproductive, lifestyle, and anthropometric risk factors for cancer in elderly women. Cancer Epidemiol Biomark Prev 22:681-687

74. Laake I, Thune I, Selmer R et al (2010) A prospective study of body mass index, weight change, and risk of cancer in the proximal and distal colon. Cancer Epidemiol Biomark Prev 19:1511-1522

75. Prentice RL, Shaw PA, Bingham SA et al (2009) Biomarker-calibrated energy and protein consumption and increased cancer risk among postmenopausal women. Am J Epidemiol 169:977-989

76. Jee SH, Yun JE, Park EJ et al (2008) Body mass index and cancer risk in Korean men and women. Int J Cancer 123:1892-1896

77. Song YM, Sung J, Ha M (2008) Obesity and risk of cancer in postmenopausal Korean women. J Clin Oncol 26:3395-3402

78. Thygesen LC, Gronbaek M, Johansen C et al (2008) Prospective weight change and colon cancer risk in male US health professionals. Int J Cancer 123:1160-1165

79. Samanic C, Chow WH, Gridley G et al (2006) Relation of body mass index to cancer risk in 362,552 Swedish men. Cancer Causes Control 17:901-909

80. Rapp K, Schroeder J, Klenk J et al (2005) Obesity and incidence of cancer: a large cohort study of over 145,000 adults in Austria. Br J Cancer 93:1062-1067

81. Ford ES (1999) Body mass index and colon cancer in a national sample of adult US men and women. Am J Epidemiol 150:390-398

82. Singh PN, Fraser GE (1998) Dietary risk factors for colon cancer in a low-risk population. Am J Epidemiol 148:761-774

83. Lee IM, Paffenbarger RS Jr (1992) Quetelet's index and risk of colon cancer in college alumni. J Natl Cancer Inst 84:1326-1331

84. Martinez ME, Giovannucci E, Spiegelman D et al (1997) Leisuretime physical activity, body size, and colon cancer in women.
Nurses' Health Study Research Group. J Natl Cancer Inst 89:948-955

85. Aune D, Vieira AR, Chan DS et al (2012) Height and pancreatic cancer risk: a systematic review and meta-analysis of cohort studies. Cancer Causes Control 23:1213-1222

86. Aune D, Navarro Rosenblatt DA, Chan DS et al (2015) Anthropometric factors and endometrial cancer risk: a systematic review and dose-response meta-analysis of prospective studies. Ann Oncol 26:1635-1648

87. Gunnell D, Okasha M, Smith GD et al (2001) Height, leg length, and cancer risk: a systematic review. Epidemiol Rev 23:313-342

88. Ahrens EH, Jr, Blankenhorn DH, Hirsch J (1956) Measurement of the human intestinal length in vivo and some causes of variation. Gastroenterology 31:274-284

89. LeRoith D, Roberts CT Jr (2003) The insulin-like growth factor system and cancer. Cancer Lett 195:127-137

90. Berkey CS, Colditz GA, Rockett HR et al (2009) Dairy consumption and female height growth: prospective cohort study. Cancer Epidemiol Biomark Prev 18:1881-1887

91. Rogers I, Emmett P, Gunnell D et al (2006) Milk as a food for growth? The insulin-like growth factors link. Public Health Nutr 9:359-368

92. Perkins JM, Subramanian SV, Davey SG et al (2016) Adult height, nutrition, and population health. Nutr Rev 74:149-165

93. Batty GD, Shipley MJ, Gunnell D et al (2009) Height, wealth, and health: an overview with new data from three longitudinal studies. Econ Hum Biol 7:137-152

94. John BJ, Irukulla S, Abulafi AM et al (2006) Systematic review: adipose tissue, obesity and gastrointestinal diseases. Aliment Pharmacol Ther 23:1511-1523

95. Giovannucci E (2007) Metabolic syndrome, hyperinsulinemia, and colon cancer: a review. Am J Clin Nutr 86:s836-s842

96. Pais R, Silaghi H, Silaghi AC et al (2009) Metabolic syndrome and risk of subsequent colorectal cancer. World J Gastroenterol 15:5141-5148 